\title{
The Research Summary of the Origin of Chinese Characters Based on the Ancient Chinese Characters and Dongba Characters Comparative Perspective*
}

\author{
Lan Wang \\ Kunming University of Science and Technology \\ Kunming, China
}

\begin{abstract}
Origin of Chinese characters is an important content of Chinese origins of civilization. Firstly, the mainstream view of the origin of Chinese characters was the theory of hieroglyph referred to as the monism in Chinese academia. And then, dualism, trichotomy and the discussion presents the development trend of pluralism. For studying the original form of Chinese characters, drawing a comparison between ancient Chinese characters and Dongba characters has important value. Now there are still deficiencies in studying the origin of Chinese characters. The suggestion is that this study should be based on a more systematic and comprehensive comparative analysis. Try to generalize the origin of Chinese characters characteristics, and to add a few links, it may be one development trend of future research in this field.
\end{abstract}

Keywords-Ancient Chinese characters; Dongba characters; compare; origin of Chinese characters

\section{INTRODUCTION}

Origin of Chinese characters is an important content of Chinese origins of civilization. This problem has been concerned by Chinese scholars for a long time. In China, study of the origin of Chinese characters has a history of more than two thousand years, dating back to the warring states period. In Chinese ancient records, there were several legends about the origin of Chinese characters, which were about of keeping records by notching wood or tying knots, and Changjie creating Chinese characters, and Chinese characters derived from Bagua.

\section{The CURRENT RESEARCH OF THE ORIGIN OF CHINESE CHARACTERS}

\section{A. The Current Research}

Firstly, the mainstream view of the origin of Chinese characters was the theory of hieroglyph referred to as the monism in Chinese academia. And then, dualism, trichotomy, and the discussion presents the development trend of pluralism.

The main representative who supported the theory of hieroglyph was Lan Tang. He puted forward the concept of "hieroglyph" in the book of "An introduction to the ancient

*Sponsor: Kunming University of Science and Technology Humanities and Social Science Research Project (Number skpyyb201622). philology"(1935), he said "the true original characters should be hieroglyphs", "Hieroglyphs evolved from the characters' drawing..."[1]. This kind of view was referred to as monism because it was that Chinese characters originated from one source.

Monism could not perfectly solve the problem of the origin of Chinese characters, especially to the problem whether primitive inscription on pottery is Chinese characters or not. With the enrichment of archaeological materials, Chinese academia gave up monism and tried from even more perspectives to explore the source of Chinese characters.

The main representative who supported the theory of dualism were Mo ruo Guo and Jian fang Yang who thought Chinese characters originated from pictographic and selfexplanatory these two systems. In the article of "The dialectical development of ancient characters" (1972), Mo ruo Guo said "Chinese characters originated from pictographic and self-explanatory these two systems[2]."In the article of "The dualism of the origin of Chinese characters"(1981), Jian fang Yang said "There were two different writing systems in the ancient Yellow River basin, that were self-explanatory and pictographic characters, $\cdots . .$. about in the period of Longshan culture (the Cheng zi ya ruins in Shandong province ) the self-explanatory characters entered the area of Shandong province, meanwhile the pictographic characters entered Central Plains, and then these two systems blended together to form inscriptions on bones or tortoise shells of the Shang Dynasty [3]."

The main representative who supported the theory of trichotomy were Ning sheng Wang. In the article of "From keeping primitive records to the invention of characters" (1981), he said "Characters was guided by three kinds of chronicle methods, rather than only evolved from the characters' drawing [4]." Three kinds of chronicle methods were that keeping records by article, symbol and the characters' drawing.

The main representative who supported the theory of pluralism were Ze ling Yang and Bao jia Li. In the article of "The pluralism of the origin of Chinese characters" (1990), Ze ling Yang said "We argue that the pluralism of the origin of Chinese characters is the same as other 
independently developed ancient writing systems in the world [5]. "In the article of "The pluralism of the origin of human characters" (1995) and "The pluralism of the origin of human characters(sequel)" (1996), Bao jia Li said "The origin of human characters is not single [6] ", "Ancient Chinese characters originated from the characters'drawing, absorbing the carved symbols", "The origin of human characters may be multivariant, due to the necessity of development of human civilization and the contingency of historical opportunity [7]."

\section{B. Conclusion}

In conclusion, about the origin of Chinese characters, there are so many theories and genres that there are not yet an unified view in academic circles. We tend to think of the pluralism of the origin of Chinese characters as true. We believe that the current research is not perfect, because the feature of the origin of Chinese characters has not been revealed enoughly, and some of the links are not clear.

\section{RETHINKING}

If the research of the origin of Chinese characters has been always carried out in the circle of Chinese ontology, It can be difficult to draw a clear conclusion. We might as well jump out of the circle of Chinese ontology, and carry out a research by drawing a comparison between ancient Chinese characters and other characters. In fact, some scholars have already one step ahead, with the beginning of the study of comparative characters, and they have made plentiful and substantial achievements, one of them is Yuan lu Wang. We were inspired by Yuan lu Wang, and we followed his research ideas, then we consider that we can study the origin of Chinese characters by drawing a comparison between ancient Chinese characters and Dongba characters.

Dongba characters is ancient writing used by Dongba priests of Naxi nationality, which is belong to ideographicphonetic writing just like ancient Chinese characters. Naxi ancestors "originated from the ancient times, dwelling in Hehuang region of northwest China, Qiang people migrated southward to the upper reaches of Minjiang River, and southwest toward the Yalong River Basin, then westwards to the upper reaches of Jinsha River [8]", after a long period of migration and reproduction. As the ancestors of Han nationality, Huaxia nationality was made up of ancient Qiang tribes, ancient barbarian tribes, ancient Rong tribes, ancient Yi tribes and ancient Yue tribes. Ancient Qiang tribes was one of the ingredients of Chinese ethnic fusion. Therefore the historical origins between Naxi ancestors and Han nationality is close. Their languages both belong to sinotibetan, the creation of the two characters is basically the same, so It provides a possibility for the comparison of ancient Chinese characters and Dongba characters.

"Inscriptions on bones" is recognized as the earliest Chinese characters mature system. From the perspective of the development history of characters, Dongba characters is more primitive than inscriptions on bones. Being a mature system of characters, Inscriptions on bones is a wordphonographic writing system, and yet Dongba characters is still in the stage of development from early stage writing to ideographic-phonetic writing. In the history of development of writing there is a number of important difference between the two characters. As De kuan Huang's "A exploration of the origin of echoism"(1986) said, "Naxi hieroglyphs is in the stage of development that it is on the eve of getting rid of the primitive stage and becoming a mature system[9]." For studying the original form of Chinese characters ,drawing a comparison between ancient Chinese characters and Dongba characters has important value.

\section{THE CURRENT RESEARCH OF COMPARISON BETWEEN ANCIENT CHINESE CHARACTERS AND DONGBA CHARACTERS}

\section{A. The Current Research}

On the origin of Chinese characters based on the ancient Chinese characters and Dongba characters comparative perspective, related research is not much.

Earlier researcher is Shan guo Jiang, whose"The origin and its structure of Chinese characters"(1930) [10] introduced domestic minorities characters and foreign ancient national characters into the study of origin of Chinese characters, and It came to a conclusion that the occurrence of world's national characters follows the common rule.

Xi gui Qiu's “A preliminary exploration of the formation of Chinese characters"(1978) [11] through the comparison of some characteristics of Chinese characters and Dongba characters, speculated that some features of ancient Chinese characters may exist, and draw a lot of valuable conclusions.

Ning sheng Wang's "From keeping primitive records to the invention of characters"(1981) [12] discussed the origin of characters by the analysis of the minorities writing material, and proposed a theory of trichotomy.

Guo yu Fang's “Spectrum of Naxi hieroglyphs” (1981) [13] consulting the six categories of Chinese characters, propounded an idea of the ten categories of Dongba characters, and compared the two characters in the aspect of word-formation method.

Jing Li's "A comparative study of Naxi Dongba characters with inscriptions on bones"(1983) [14] was the first thesis which compared pictographic, echoism and phonetic loan characters of the two characters, making a valuable attempt.

De kuan Huang's "A exploration of the origin of echoism"(1986) [15] explored the origin of echoism, through the comparison of echoism of Chinese characters and Dongba characters.

Having the plentiful and substantial achievements, Yuan lu Wang published a series of papers such as "The comparison of phonetic loan characters of Dongba characters and ancient Chinese characters and its value in the history of characters" (1987) [16], "The comparison of echoism of Dongba characters and Chinese characters"(1987)[17], "The different origin of Naxi Dongba characters and Chinese characters"(1987) [18], and he found some characteristics of early Chinese characters by comparing the two characters. 
His books "The comparison of ancient Chinese characters and Dongba characters"(1988) [19], "Comparative philology"(2001)[20] speculated the overview of the early Chinese characters through the comparison of different characters.

Recently, Jiang wen Luo's "Discussing problems in the study of origin of Chinese characters according to Yunnan minorities writing material" (2014) [21] provided some evidences for the research on origin of Chinese characters, through the analysis of yunnan minorities writing material.

\section{B. Problem and Suggestion}

To sum up, on the origin of Chinese characters based on the ancient Chinese characters and Dongba characters comparative perspective, existing research is not enough. From the review of previous studies, we found that there are three deficiencies.

1) The existing research is most of the comparison of the individual phenomenon, but lack of systematic comparison: Actually, what is generally referred to as "ancient Chinese characters" includes many materials. But researchers tend to choose one individual or part of the materials to compare, this leads to the lack of systemicness. We think that materials of ancient Chinese characters should be collected according to the classification, which includes primitive inscription on pottery, inscriptions on bones and inscriptions on ancient bronze objects. We can compare these three kinds of materials respectively with Dongba characters.

The first kind of material is primitive inscription on pottery, some of which are controversial materials such as carved symbols and characters' drawing. Whether they are characters or not, it remains controversial in the academic community. Some scholars considered that primitive inscription on pottery is not ture characters, because it does not have the founction of recording language. However, quite a part of scholars thought that the nature of characters of primitive inscription on pottery should be identified, since it has so obvious characters feature that many character patterns of primitive inscription on pottery are inherited by Inscriptions on bones. We must form the breakthrough in theory of the understanding of it, because we can not evade this problem when we study on origin of Chinese characters. We tend to think of the nature of characters of primitive inscription on pottery as true. In the history of development of writing, primitive inscription on pottery is in more earlier stage than Dongba characters. The comparation of this two characters should focus on both similarity and difference, because whether similarity or difference, may help to conjecture the feature of the origin of Chinese characters.

The second kind of material is Inscriptions on bones, which is recognized as the earliest Chinese characters mature system. In the history of development of writing, Dongba characters is in a bit earlier stage than Inscriptions on bones. The comparation of this two characters should focus on difference rather than similarity. We should see the rule reflected from their difference, and conjecture the feature of Chinese characters.
The third kind of material is inscriptions on ancient bronze objects, which is a mature system, but has outstanding pictographic characteristics. In the history of development of writing, Dongba characters is in more earlier stage than inscriptions on ancient bronze objects. The comparation of this two characters should focus on difference rather than similarity. This difference is due to two characters have not only similar nature but also successively relationship on the stage. On the analysis of the difference is more conducive to trace the characteristics of Chinese characters from the origin to the mature stage.

These three kinds of materials is representative of ancient Chinese characters from embryonic origin to mature period. With the integrality of materials, it is ensured that research will be systematic.

2) Existing research is not comprehensive enough: Existing research mainly make a comparison in two respects that include word-formation method and Symbol form, but less involved in other aspects. We think that comparison may be made in four respects of characters configuration, word-formation method, word-formation psychology and word-formation mechanism.

The first aspect is characters configuration, which is also called symbol form. Characters configuration is the external form of the nature of a writing system, and also an important measure of characters development. Naxi hieroglyphs is in the stage of development that it is getting rid of the primitive stage and becoming a mature system, therefore its characters configuration keep a large number of the characteristics of the primitive drawing. From primitive inscription on pottery to inscription on bones, and then to inscriptions on ancient bronze objects, with the development trend of symbolization and simplification, ancient Chinese characters configuration is bound to be near typical symbol shape characteristics. Both Dongba and ancient Chinese characters each have their own distinct stage characteristics.

The second aspect is word-formation method, which is the six categories of Chinese characters, the traditional Chinese theory used to analysis structure of Chinese characters. The six categories are pictographic characters, self-explanatory characters, associative compounds, echoism, mutually explanatory and phonetic loan characters. Both Dongba and ancient Chinese characters are ideographicphonetic writing, but Dongba characters is still in the stage of pictographic writing, and yet inscription on bones is in the stage of phonetic -pictographic writing. These two characters mainlye use the word-formation method of pictographic characters, associative compounds, echoism and phonetic loan characters, but each have their own characteristics. Individual differences of the two can show unique personality and style of all kinds of ideographic-phonetic writings, and common characteristics of the two can show pervasive nature of general ideographic-phonetic writing.

The third aspect is word-formation psychology, which refers to analyze the psychological characteristics of the ancient people creating characters from the social and cultural background. For example, there are independent 
entity of pictographic characters and complex pictographic characters in both Dongba and ancient Chinese characters. From independent entity of pictographic characters to complex pictographic characters, from primitive concrete thinking to abstract concept thinking, it shows that conceptual work of human beings gradually become complicated and abstract.

The fourth aspect is word-formation mechanism, which refers to analyze the cause and motive of origin of characters from the perspective of historical development. Characters is symbol system recording language, and language is the main social communication tools, so the occurrence of characters depends on the development of the society.

Ancient Chinese characters and Dongba characters occur in different time, the history of the development is also different. Drawing a comparison between ancient Chinese characters and Dongba characters is helpful to study the original form of Chinese characters. Respectively comparing Dongba characters with primitive inscription on pottery, Inscriptions on bones and inscriptions on ancient bronze objects, we may deduce common characteristics of different development phases of characters.

3) Third, existing research is not in-depth enough: About the origin of Chinese characters, conclusion of existing research focuses on an overview of the macro level, and lack of intensive analysis on the feature of original Chinese characters and the links of occurrence.

We think that there are symbolic characters, ideographic characters, phonetic characters , and so on, in the beginning phase of Chinese characters. The origin of Chinese characters is multivariant. The study should be based on a more systematic and comprehensive comparative analysis. Try to generalize the origin of Chinese characters characteristics, and to add a few links, this may be one development trend of future research in this field.

\section{REFERENCES}

[1] Lan Tang, "An introduction to the ancient philology", Laixunge Bookstore, 1935.

[2] Mo ruo Guo, "The dialectical development of ancient characters", Acta Archaeologica Sinica, vol. 1,1972.

[3] Jian fang Yang, "The dualism of the origin of Chinese characters", Studies of Chinese Language, vol. 3, 1981

[4] Ning sheng Wang, "From keeping primitive records to the invention of characters", Acta Archaeologica Sinica, vol. 1,1981.

[5] Ze ling Yang, "The pluralism of the origin of Chinese characters", Journal of Zhengzhou University, vol. 4,1990.

[6] Bao jia Li, "The pluralism of the origin of human characters", Journal of Liberation Army institute of foreign languages, vol. 6,1995.

[7] Bao jia Li, "The pluralism of the origin of human characters(sequel)", Journal of Liberation Army institute of foreign languages, vol. 1,1996.

[8] Guo yu Fang, "Spectrum of Naxi hieroglyphs", Yunnan People's Publishing House, 1981.

[9] De kuan Huang ,"A exploration of the origin of echoism ", Journal of Anhui educational college,vol.3,1986.

[10] Shan guo Jiang, "The origin and its structure of Chinese characters", The Commercial Press, 1930.
[11] Xi gui Qiu, "A preliminary exploration of the formation of Chinese characters", Chinese Language,vol. 3, 1978.

[12] the same as[4]

[13] the same as[8]

[14] Jing Li, "A comparative study of Naxi Dongba characters with inscriptions on bones", Yunnan Social Science,vol. 6, 1983.

[15] the same as[9]

[16] Yuan lu Wang, "The comparison of phonetic loan characters of Dongba characters and ancient Chinese characters and its value in the history of characters ", Journal of XuZhou Normal College, vol. 2, 1987.

[17] Yuan lu Wang, "The comparison of echoism of Dongba characters and Chinese characters", Journal of Central Academy of National Minorities,vol.5,1987.

[18] Yuan lu Wang, "The different origin of Naxi Dongba characters and Chinese characters", Journal of Yunnan Nationalities Institute,vol. 1, 1987.

[19] Yuan lu Wang, "The comparison of ancient Chinese characters and Dongba characters",East China Normal University Press, 1988.

[20] Yuan lu Wang, "Comparative philology",Guangxi Education Press, 2001.

[21] Jiang wen Luo, "Discussing problems in the study of origin of Chinese characters according to Yunnan minorities writing material", Journal of Chinese Linguistics, vol. 16, 2014. 